EIECTRO WMANTIC FIELDS IN AN AXIAL SYRIETRIC WAVEGJIEE WTTI VARLABLE CROSS SECTICN *

\title{
S. Wheifets
}

Stanford linear Aecelerator Center, Sturford thiversity, Stanford CA 94505 USi.

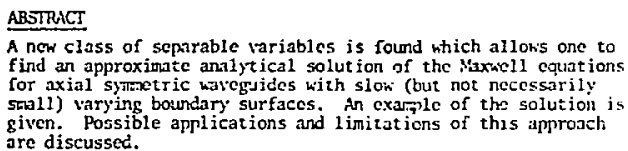

There are many problens the soJution of which can be reduced to a wach siafiler cni if only the cigenfunctions of the corresponding equations were found. The cnly one known ana. Jytical solution of the inxell equations for the space inside an axial sytgritic wideguide js tlic solution for the wateguide with a constant cijcular cross sec:ion. Thi solutions for a stephise constant cross section katequide can be obtained by prorerly ratching the solut jous for judivjdual steps. This procdure is time and labor consuming. The cximi ld of such an approach for a stephise perjocic structure is given in hork. "l

Exact aralytical solution for a ka eguide with variable cross section is hot buvr

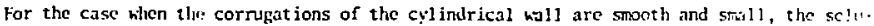
tion can be obtained by weans of perturbation theory ${ }^{2,3)}$ This nesthot was recin: 1 y alyl it.. to prohler.s of fjnding, the mike fields induced by a current moving on axist and aff axis." of a corruñated pipe.

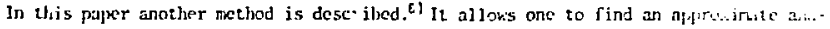

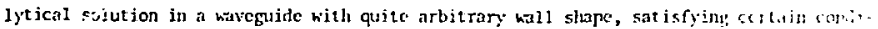

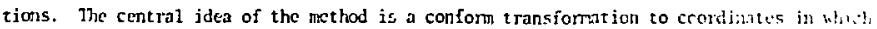
the beudary curve appears to be a straight line. In the neh orthopenal curvilunear en-ydjnates the verjables are separable even in the vector kave cquation. Nalopiculy to $:^{-i-}$ indric waveguide with constant cross section $7 \mathrm{M}$ and TE modes are intraltect. Next stef is then to satjsfy the boundary conditions fer the electromsporic field inside the fipe atul to find the eigenvalues (the frequeneics and the propagation functionsj.

An exarple of the application of the derived methat as well as tia contarisan ni the calculations with the results of deasurcments taken from the paper ${ }^{2}$ erc give:..

Let us coisjder the following dependr..jes of the rectangulat conrdinatee $x, y, z$ ת curvilinear coordinates $0, \theta, 0$ :

$$
\begin{aligned}
& x=A\left(p+\sum_{\pi} a_{n} \operatorname{shn} \cos n o\right) \cos \theta \\
& y=A\left(p+\sum_{i=} a_{n} \sin n o \cos n o\right) \sin \theta \\
& z=A\left(0+\sum_{\pi} a_{n} \operatorname{ch} n \sin \pi \sigma\right)+z_{0}
\end{aligned}
$$

Fion (1) and (2) we get for the radius in the plane $z=$ const

$$
r=A\left(0+\sum_{n} a_{n} \text { sh no } \cos n c\right)
$$

- hork sujported by the Department of Energ;, contract DE-AC05-76SF00515.

(Contributed to the $x 1$ Internstional Conference on lligh lievgy Accelorptors, Ches, Consta, Siritzerland, Jul) $7-11,1980$. 
Symbol $\sum_{n}$ means either surmation or integration over n. Gonerally speaking, one needs to distinguish between covariant and contravariant components of any vector $\vec{F}$. To avoid ehis, I will use only projections $F_{1}, F_{2}, F_{3}$ of any vector $\vec{j}$ on a base systen of unit vectors $\vec{i}_{1}$, $\vec{i}_{2}, \vec{i}_{3}$, Using Equations $(1,2,3)$, it is casy to 1 ind the mettic tensor $s_{i j}$.

$$
B_{i j}=0, \quad i \neq J
$$

proves the orthogonality of the coordinate system, $0, \theta, 0$. From (4) follows that $r=0$ for $D=0$. Constant $z_{0}$ in (3) allows tire choice of any plane $z=$ const for placing there the origin $p=0=0$. If we put ${ }^{2} 0=0$, then the origin will be in the piane where the eross section has the maximum. The constant $A$ is an arbitrary seale factor with the dimension of the length. The $0, \theta$ and 0 are dimensiontess. In the $J$ imit $a_{n} \rightarrow 0,0, \theta$ and $a$ go into usual cylindrical coordinstes $r, \theta, z$. The constants $a_{n}$ are dimensionless arbitrary quantities limited only by a natural condition $r>0$ e nthere. The case $r=0$ at sone value of o should be considered separately (it represents a ciosed axial symetric cavicy of an arbitrary shape).

The metrjc coefficients $h_{i}=\sqrt{g_{i j}}$ are very complicated and do not give the possibility to solve the corresponding Alanell ceputions. They becanc raxh sinpler in the 1 imit

$$
N_{p}<1 ;
$$

where $N$ is the post sufficjent birmonic number in the expansion (A). If (6) is fuifilyed, then

$$
\begin{aligned}
h_{j}= & h_{3}=h(a), \\
& h_{2}=p h(0), \\
h(\sigma)= & n\left(1+\sum_{n} a_{n} u \cos n n\right)
\end{aligned}
$$

where

For the coordinate transformation we get

$$
\begin{aligned}
& r=\operatorname{oh}(\sigma) \\
& z=\sigma^{\sigma} h\left(\sigma_{1}\right) d \sigma_{1} \\
& r=\rho i(z)
\end{aligned}
$$

If one chooses the function $f(z)$ in such 7 vay as to describe the shape of the wall in the plane $\theta=$ const, then $0=o_{\max }=$ const on $t .2$ koundary. The physical meaning of the condition (6) dejends on the actual walue of the coefficients in for, in otlier words, on actuat shape of the Loundiry). But, in the casc wierc all $a_{n}$ are sinall, the meaning can be understood casily, In this case, $z$ Aa,

$$
\begin{aligned}
r_{\text {boundar, }} & =o_{\max } f(z)=p_{m 1 x} h(z / N) \\
& =A_{\max }\left(1+\sum_{n} a_{n} n \cos n z / A\right)
\end{aligned}
$$

and we see that $A=L / 2 \pi$, where $L$ is the characteristic length of the change of the function $f(2)$ (the period for a periodic function). Now, take the average of equation (13) over $z$ :

ot

$$
\begin{aligned}
& \left\langle r_{\text {baundary }} \equiv b=A_{\text {max }}=l_{\text {rax }} / 2 x\right. \\
& D_{\max }=2 \mathrm{nb} / \mathrm{L} \text {. }
\end{aligned}
$$

where $b$ has the meaning of the average pipe radius. The condition (6) now gives

Since o changes in the linits

$$
\begin{aligned}
& b<L / 2 \pi \mathrm{N} \\
& 0 \leq 0 \leq p_{\max }
\end{aligned}
$$

the condition (6) is fulfilled for all the values of $p$, and the afproximation (7)-(9) is valid everytere inside the pipe. 
Since expansion (9) contains only cos'nes, not all shapes of the bouniary can be treaced in this way. Namely, we can consider only such boundary curves $\mathrm{T}=\mathrm{r}(\mathrm{z})$, which al low the representation in the forn (10), (11) with h(o) symetric

$$
h(-\sigma)=h(\sigma)
$$

For the sake of completeness, it is useful to mkntion al so the restrjiction of the method b: axial symetric waveguides only.

Let us confine ourselves to the case hten the matis inside the woveruide is a ratum. The generaljzatjon to a homgeneous dielectric nedia is straightformard. In the aisence of charges and currents, the fields can be desined'in tens of tho vector functions, $\vec{i}$ and $\vec{\pi}$ (the liertz vectors); each one of them satisfies homogelicous vector wive cquation. Since the coefficients of this equation are constant it is possibic 20 deal with cach $t$ isme Fourier lamonic of $\overrightarrow{i t}$ and $\vec{\pi}$ separately:

$$
\begin{aligned}
& \vec{\pi}=\int \vec{\pi}_{\omega} i^{-i \omega t} d \omega \\
& \vec{\pi}=\int \vec{\pi}_{\omega}^{*} a^{-i \omega t} d_{\omega}
\end{aligned}
$$

Then, $\vec{\pi}_{\omega}$ and $\vec{\pi}_{\omega}^{*}$ satisfy the equations:

$$
\begin{aligned}
& \nabla^{2} \pi_{\omega}+k^{2+\pi_{\omega}}=0, \\
& \nabla^{2} \pi_{\omega}+k^{2+\pi_{\omega}}=0,
\end{aligned}
$$

where

$$
\mathbf{k}=\omega / c .
$$

The differential operator $\nabla^{2}$ here is deisnid by:

$$
\nabla^{2} \vec{\pi}_{\omega j}=\nabla \cdot \nabla \vec{\pi}_{\omega}-\nabla \times \nabla \times \vec{\pi}_{u}
$$

In all the further formulac, I onit the subseript $w$ by fourier components of the vector functions.

The ficlds can be found, provided $\vec{\pi}$ and $\vec{\pi}$ are known:

$$
\begin{aligned}
& \vec{E}^{(1)}=\nabla \times \nabla \times \vec{\pi} \\
& \vec{j}^{(1)}=-i 1 \nabla \times \vec{\pi} \\
& \vec{E}^{(2)}=i k \nabla \times \vec{\pi} \\
& \vec{H}^{(2)}=\nabla \times \nabla \times \overrightarrow{\|}
\end{aligned}
$$

Vectors $\overrightarrow{\mathrm{E}}^{(1,2)}$ and $\vec{H}^{(1,2)}$ al so satisfy equat ion analogous to (21).

In coordinates $p, \theta, 0$ and with the metrie corfficjents $(7),(8)$ the project jors of th equation (21) on axes $\vec{i}_{i}$ have the rolloxing form:

$$
\begin{aligned}
& \frac{1}{h^{2}} \frac{\partial}{\partial \theta} \frac{1}{\partial} \frac{\partial}{\partial p} \rho \pi_{1}+\frac{1}{\partial^{2} h^{2}} \frac{\partial^{2} \pi_{1}}{\partial \theta^{2}}+\frac{1}{h^{2}} \frac{\partial}{\partial \theta} \frac{1}{h} \frac{\partial}{\partial \theta} h n_{1}+k^{2} \pi_{1}+\frac{1}{h^{2}} \frac{\partial}{\partial \theta} \frac{1}{\partial \theta} \frac{\partial \pi_{2}}{\partial \theta} \\
& -\frac{1}{\partial^{2} h^{2}} \frac{\partial}{\partial \rho} \cdot \frac{\partial \pi_{2}}{\partial \theta}+\frac{1}{h^{4}} \frac{\partial^{2}}{\partial a \partial D} h^{2} \pi_{3}-\frac{1}{h^{2}} \frac{\partial^{2} \Pi_{5}}{\partial 0 \partial p}=0 \\
& \frac{1}{h^{2}} \frac{\partial}{\partial o} \frac{1}{\partial} \frac{\partial}{\partial \rho} \rho \pi_{2}+\frac{1}{p^{2} h^{2}} \frac{\partial^{2} \pi_{L}}{\partial \theta^{2}}+\frac{1}{h^{2}} \frac{\partial}{\partial 0} \frac{1}{h} \frac{\partial}{\partial o} h \pi_{2}+h^{2} n_{2}+\frac{1}{h^{2} p^{2}} \frac{\partial^{2} \rho r_{1}}{\partial \sigma \partial c} \\
& -\frac{1}{h^{2}} \frac{\partial}{\partial t} \frac{1}{p} \frac{\partial t_{1}}{\partial \theta}+\frac{1}{p h^{4}} \frac{\partial}{\partial \sigma} h^{2} \frac{\partial n_{3}}{d \theta}-\frac{1}{\partial h^{2}} \frac{\partial^{2} n_{3}}{\partial \sigma \partial \theta}=0
\end{aligned}
$$




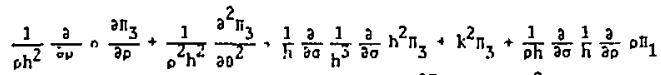

$$
\begin{aligned}
& -\frac{1}{\rho h^{3}} \frac{\partial}{\partial \rho} \rho \frac{\partial}{\partial \sigma} h \pi_{1}+\frac{1}{i: 0} \frac{\partial}{\partial \sigma} \frac{1}{h} \frac{\partial \pi_{2}}{\partial \theta}-\frac{1}{\operatorname{ch}^{3}} \frac{\partial^{2}}{\partial \sigma \partial \theta} h \pi_{2}=0
\end{aligned}
$$

The same sysiem of equations holds al so for the vector $\vec{i}$.

To solve cquations (28)-i;o) we jook for the so?uticn in the folloking form:

$$
\begin{aligned}
& \pi_{1}=J_{m}^{\prime}(\lambda s) F_{1}(0) e^{j \mu \rho} \\
& \pi_{2}=j m \frac{J_{m}\left(\lambda_{0}\right)}{\rho} F_{2}(\sigma) e^{j \pi \theta} \\
& \pi_{3}=\lambda J_{m}(\lambda p) F_{3}(\sigma) c^{j m \theta}
\end{aligned}
$$

A prime from tow on means the differentiation with respect to o if it is appliced to a

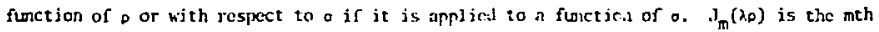
order Bessel fumction, $\lambda$ is an arbitran; constant, and $m$ is any positive $c$. negative integer is luding zero. The runction $r_{1}, F_{2}, F_{3}$ is unhohn up to the tink and shold be chosen in suih a way that the functions (31)-(33) satisfy eqpations (28)-(30).

The llertz vector $\overrightarrow{\|}(31)-(32)$ allows one to find the electric $\vec{E}^{(1)}$ and ragnetic $\vec{H}^{(1)}$ ficle : from s.spressjons (24) and (25). Using tl : expressions for differential (pe ators in our coordinates $\rho, 0,0$, one gets:

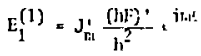

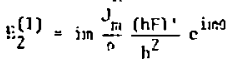

$$
\begin{aligned}
& E_{3}^{(1)}=\lambda^{2} J_{m} \frac{F}{h} e^{i m 0} \\
& \mathrm{H}_{\mathrm{j}}^{(1)}=\mathrm{ml}: \frac{\mathrm{J}_{\mathrm{m}}}{\mathrm{r}} \mathrm{F} \mathrm{c}^{\mathrm{im} 0} \\
& H_{2}^{(1)}=i k \cdot{ }_{m}^{1} F c^{i \pi, 0} \\
& 1 u_{3}^{(1)}=0
\end{aligned}
$$

The function $F(0)$ is a solution of the following a. at ion-

$$
F^{\prime \prime}+\frac{h^{\prime}}{h} F^{\prime}+F\left(\frac{h^{\prime \prime}}{h}-\frac{h^{2}}{h^{2}}+k^{2} h^{2}-\lambda^{2}\right)=0
$$

hic see that the third component of the menetic ficld, $\overrightarrow{l l}(1)$ is zero. Analogously to eyl indrical coordinates $\mathrm{he}$ can call the fields $\overrightarrow{\mathrm{E}}^{(1)}$. $\overrightarrow{\mathrm{H}}^{(1)}$ transverse ragnet ic modes (TN modes). One should remember, of course, that the field $\vec{H}^{(1)}$ is transverse to the direction $\vec{i}_{3}$, but it is not transverse to the axis of the anveguids $\vec{i}_{2}$.

The field $\overrightarrow{\mathrm{E}}^{(1)}, \overrightarrow{\mathrm{H}}^{(1)}$ should satisfy al so the bourdary conditions. That can be achieved by proper choosing of the value of the constant $\lambda$. Namely, it impics:

or

$$
\begin{aligned}
& J_{m}\left(\lambda \rho_{n a x}\right)=0 \\
& \lambda_{m k}=v_{m k} / o_{\max }
\end{aligned}
$$

where $v_{m k}$ is the kth root of equation $J_{m}(x)=0$, 
The second independent set of electrowanetic riclds inside the waveguide can be obtained from the sank veetor $\vec{n}(31)-(33)$ by means of equations (26), (27). Ke can call these modes transvorse electric nodes (Tl: modes) since $\mathrm{E}_{3}^{(2)}=0$. To sat isfy the bundary conditions for the TE modes, the value of tl:e constant $\lambda$ should be chosen in such a way that

$$
J_{\pi}^{\prime}\left(\lambda \rho_{\max }\right)=0
$$

or

$$
\lambda_{\mathrm{mk}}=\dot{v}_{\mathrm{mk} /{ }_{\text {IRiLx }}} \text {, }
$$

where $\bar{v}_{m k}$ is the kth root of equation $\mathrm{J}_{1 n}^{\prime}(x)=0$.

Let us 10ok more closely on the o-deperdence of the riclus $\vec{E}$ a'd $\vec{H}$. It is described by the function $F$ which satisfies cquation ( 40$)$. Since $z$ is the function of o only, ne can cone back from the o varjable to the 2 variable.

$$
\begin{aligned}
& d z=l i d \sigma \\
& h^{\prime}=\| \frac{d h}{d \bar{z}}
\end{aligned}
$$

lt is convenjent at the sane tiuc to change the function $F(0)$ to a new function $u$ :

$$
u(z)=F(0) \cdot \ln (\sigma),
$$

where $j$ is is unerstond that in the right-hand side of expression (45) o should be substituted by the function a(z) from (11). 7hen, from the equation (40) he get the following cquation for the function u:

$$
d^{2} u / d z^{2}+\left(v^{2}-v^{2} / r_{b}^{2}\right) u=0 \text {, }
$$

where $r_{b}=r_{b}(z)$ is the boundary cusce. For Thi moles, we eet not:

$$
\begin{aligned}
& E_{l}^{(l)}=v \cdot J_{m}^{\prime} \frac{u^{\prime}}{r_{b}} c^{i n g},
\end{aligned}
$$

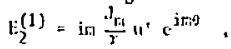

$$
\begin{aligned}
& \mathrm{E}_{3}^{(1)}=v^{2} \frac{\mathrm{J}_{\mathrm{n}}}{r_{\mathrm{b}}^{2}} \mathrm{u} \mathrm{e}^{i n n} \text {, } \\
& H_{j}^{(1)}=\operatorname{mk} \frac{j}{j} u e^{i m o} \text {, } \\
& \mathrm{H}_{2}^{(1)}=\text { ik } \frac{\nu}{\mathrm{r}_{b}} \cdot \mathrm{IJ}_{\mathrm{m}} u \mathrm{c}^{\mathrm{jmg}} \text {, } \\
& \mathrm{H}_{3}^{(1)}=0 \text {, }
\end{aligned}
$$

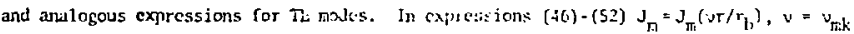

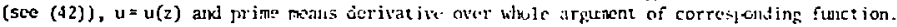

In the limjt of the constant cross section waveguide $r_{b}=$ ronst and the solut ion of equat ion (46) is

$$
u=\exp \left( \pm \sqrt{n^{3} \cdot \overline{\lambda^{2}} z}\right)
$$

he can call $u(z)$ a propagation function. Whes $r_{1}(z)$ is a periodic function, cquation (46) becones the Hill equation. Thic sulution of it defines positions and widths of slopbands is well as the phase velocjty of the kave in passbands. Nore detailed investigat jon of the bchavior of the propagation function can be done only for a given function $r_{h}(z)$.

1 present here an exingle of the application of the theory. The propagation floction. uff) is calculated for the walepuide with the following boundary:

$$
r_{b}=b(1+a \cos 2 \pi z / L) \text {, }
$$

where $b$ is tik average radjus or the pije's cross section, a and $L$ are the arglitude and the feriod of the boundary variatjon. The ajplicability of the theory limits the values 
of $b$ and $L$ by the condition $\rho_{\max }=2 \pi b / L<1$. The general solution of equation (46) in regions of its existance ("passbards") can be expressed in terms of the corresponding B.function of this equation: 7$\}$

where

$$
\begin{aligned}
& u(z)=u_{0} \sqrt{B(z)} e^{i \phi(z)-i_{\omega t}} . \\
& \phi(z)=\int_{0}^{2 \Delta z / L} d \xi / s(\xi)
\end{aligned}
$$

and the function $\zeta(2)=\sqrt{\beta(z)}$ is the solution of the following nonlinear equation:

$$
c^{\prime \prime}+\left(k^{2}-v^{2} / r_{b}^{2}\right)_{c}=1 / c^{3} .
$$

Out side of the passbands there"js no solution of enuation (46) ("stoiksunds"). The elcetronagnetic wave with corresponding frequencies (or values $k$ ) can not propafate in the waveguide under consideration. Inside one of the passbands we can determine the phase velocity ${ }_{p h}$ of the wave. From (55) one finds

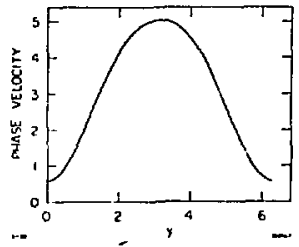

Fig. 1. The plase velocjt) of the TMol $(y=2.405)$ mode is longjtudinal coordinate $y=2 \pi 2 / 1$. The waveruide paraneters are pinax $=$ $2.87, a=0.111, K$ is sbove the stopband shokn on Fig. 2 .

$\mathrm{K}=1.03, \mathrm{vay}=3.04$. $v_{\mathrm{ph}} / \mathrm{c}=(\mathrm{L} / 2 \pi)$ a $(\mathrm{z})$.

The phase velocity is not constunt, but it is nodulated with the frequency $2 \pi / \mathrm{L}$. Figure 1 iJlustrates the dependence of the phase velocity versus the longitulinui coordinate $y=2 \pi z / L$ for different values of the paranteters $k=$ $k l / 2 \pi, p_{\max }=2 \pi b / L$ and $a$. For some problems the average (over 2) plase velocity is of interest. This guantity is relevant for example to a problem of the coupling betwetl the electromagnet ic wave pronagating in the bavegujde and a particle moving along jts axis. The relative arerag. phase velocity $v_{p h} / c$ is also given on this figure. Figue? shows dependencies of the alerage phin velocity oa the patrameter $K$ as well as posiljon and widths of the stopband.

The case $D_{m 2 x}=2.87$ is far outside the validity of the described method. It was calculated hele for the

sake of the comparison kith shown results. The crosses on Fig. ? represent the results of calculations according to second order perturbation theory and of the mearursments and are taken from the nork. ${ }^{21}$

\section{ACNOONLEILGENENS}

I am grateful to Phil Norton, Alex Chno, Perry Wilson, Karl bane and $\mathrm{C}$. Yao for helpful discussions and crjticism.

$* *$

REFEREYCFS

1) B. Zotter and K. Bane, PEP-Note 308 (1979) SLAC.

2) J. Chandezon, G. Comict and G. Roult, C. R. Acad. Sci. Paris, t.277, Serie B., p. 405 (1973).

3) D. R. Asfar and A. I1. Nayfeh, IEEE Trans, on MTT, Vol. 23, ha. 9, p. 728 (1975).

4) M. Chatatu-koul in and A. Papiernik, IEeE Trans. on k's, Vol. 26, No. 3, p. 3523 (1979).

5) p. Mrton, private commication.

6) S. Wheifets, SLAC-PUB-2547, PEP-Hote-331, tune 1980.

7) E. Courant, H. Snyter, Annals of Plyss., Vol, 3, No. 1 , p. 1 (1958): also in M. Sands, SiLC-121 (1970).

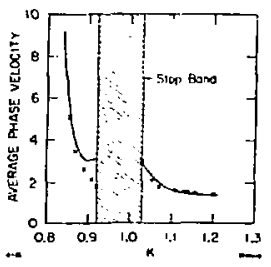

Fig. 2, The average relative phase velocity of the TMul mode versus paraneter $k=1 \cdot 1 . / 2 \pi$. The waveguide paribneters are Prax $=2.87, a=0.111$. The crosses represent the results of the calculations and pieasureanent 5 of the wark. 2)

Al tho:igh this case is outsiue the applicability region of this work method, the corresponùence is not at all bad. 